\title{
Programa Nacional de Melhoria do Acesso e da Qualidade da Atenção Básica: questões a problematizar
}

\author{
National Primary Care Access and Quality Improvement Program: issues to discuss \\ Programa Nacional de Mejoramiento del Acceso y de la Calidad de la Atención Bàásica: \\ cuestiones para debatir
}

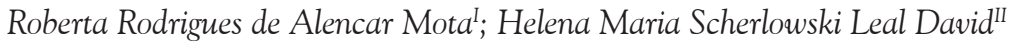

\begin{abstract}
RESUMO: Este artigo objetivou refletir sobre o Programa Nacional de Melhoria do Acesso e da Qualidade da Atenção Básica (PMAQ-AB) como estratégia atual de avaliação dos serviços de atenção básica utilizada pelo Ministério da Saúde. Destaca alguns de seus aspectos mais relevantes, tendo como norte a efetivação da implementação do Sistema Único de Saúde como política social voltada para o acesso universal e a equidade em saúde, por meio da descentralização e fortalecimento dos níveis municipais e locais de saúde. Apesar de sua recente implantação, em 2011, atualmente o PMAQ-AB encontra-se em seu segundo ciclo, o que é considerado muito precoce, visto que todo o processo nacional é bastante complexo, dispendioso e exaustivo, tanto para as equipes de atenção básica que são avaliadas quanto para a as equipes de avaliadores. Além disso, é um tempo demasiadamente curto para identificação de problemas, definição de estratégias de intervenção e mudança de realidades. Palavras-Chave: Atenção primária à saúde; diagnóstico da situação de saúde; garantia da qualidade dos cuidados de saúde; qualidade, acesso e avaliação da assistência à saúde.
\end{abstract}

\begin{abstract}
This article reflects on the National Primary Care Access and Quality Improvement Program (PMAQ-PC) as a current strategic for reviewing primary care services used by the Ministry of Health. It highlight some of its most relevant features, as they relate to effective implementation of the Unified Health System as a social policy designed to afford universal access and equity in health by decentralizing and strengthening health care at the municipal and local levels. Although introduced only recently (in 2011), the PMAQ-PC is currently in its second phase, which is considered very premature, since the entire national process is very complex, expensive and exhaustive, both for the primary care teams evaluated and the teams of evaluators. Also, it is too short a time to identify the problems, develop intervention strategies, and change conditions.

Keywords: Primary health care; health situation analysis; health care quality assurance,; health care quality, access, and evaluation.

RESUMEN: Este artículo tuvo por objetivo presentar una reflexión sobre el Programa Nacional de Mejoramiento del Acceso y de la Calidad de la Atención Primaria (PMAC-AP) como la revisión estratégica actual de los servicios de atención primaria que utiliza el Ministerio de la Salud, destacando algunos de sus aspectos más relevantes, tiendo como norte la implementación del Sistema Único de Salud - política social dirigida al acceso universal y a la equidad en salud, a través de la descentralización y fortalecimiento de los niveles de salud municipales y locales. A pesar de su reciente creación, en 2011, actualmente el PMACAP se encuentra en su segunda fase, lo que se considera muy temprano, ya que todo el proceso nacional es muy complejo, costoso y agotador, tanto para los equipos de atención primaria que son evaluados por los equipos de evaluadores. Además, es un muy corto tiempo para la identificación de problemas, definición de estrategias de intervención y cambio de realidades. Palabras Clave: Atención primaria de salud; diagnóstico de la situación de salud; garantía de la calidad de los cuidados de
\end{abstract}

\section{INTRODUÇÃO}

O ano de 2011, além da publicação de uma nova versão da Política Nacional de Atenção Básica (PNAB), também foi marcado pelo lançamento da Portaria no 1.654 do Ministério da Saúde (MS), que institui, no âmbito do Sistema Único de Saúde (SUS), o Programa Nacional de Melhoria do Acesso e da Qualidade da Atenção Básica (PMAQ-AB) e o Incentivo Financeiro do PMAQ- $\mathrm{AB}^{1,2}$. É importante ressaltar que com esse movimento do MS foi possível a pactuação e publicação de uma série de portarias e atos normativos que criaram novos programas (dentre eles o PMAQ-AB) e produziram avanços em ações e programas já existentes, viabilizando, na perspectiva de alguns autores, uma nova PNAB para o país ${ }^{3}$. O PMAQ-AB é considerado o programa que melhor sintetiza essa nova PNAB, exatamente por se articular com diversas iniciativas

estabelecendo uma relação de síntese e sinergia com quase a totalidade delas, podendo ser colocado na condição de revelador da nova PNAB ${ }^{4: 361 .}$

'Enfermeira. Doutorando do Programa de Pós-Gradução da Faculdade de Enfermagem, Universidade do Estado do Rio de Janeiro. Brasil. E-mail: robertarodriguesmota@yahoo.com.br

"Doutora em Saúde Coletiva. Professora Adjunta do Departamento de Enfermagem em Saúde Pública e do Programa de Pós-Graduação em Enfermagem, Faculdade de Enfermagem, Universidade do Estado do Rio de Janeiro. Brasil. E-mail: helena.david@uol.com.br 
Com a introdução do PMAQ-AB, o MS tenta fortalecer a qualificação das ações ofertadas no nível primário de atenção.

O conjunto de ações e atividades desenvolvidas no âmbito do PMAQ é uma das estratégias indutoras da qualidade adotada pelo MS na direção da melhoria dos resultados das ações de saúde ofertadas à população $0^{5: 15}$.

A Portaria no 1.654 é direcionada à todas as equipes de saúde da atenção básica (EAB), ou seja, as EAB contratualizadas, Equipes de Saúde Bucal e os Núcleos de Apoio à Saúde da Família (NASF). As equipes podem aderir ao PMAQ-AB desde que estejam em conformidade com os princípios da atenção básica e com os critérios definidos no Manual Instrutivo do PMAQ-AB22.

\section{O principal objetivo do PMAQ é:}

induzir a ampliação do acesso e a melhoria da qualidade da atenção básica, com garantia de um padrão de qualidade comparável nacional, regional e localmente de maneira a permitir maior transparência e efetividade das ações governamentais direcionadas à Atenção Básica em Saúde ${ }^{1: 1}$.

Entre os seus objetivos específicos, destacamos: ampliar o impacto da AB sobre as condições de saúde da população e sobre a satisfação dos seus usuários; fornecer padrões de boas práticas e organização das unidades básicas de saúde (UBS) que norteiem a melhoria da qualidade da $\mathrm{AB}$; promover maior conformidade das UBS com os princípios da $A B$; promover a qualidade e inovação na gestão da $A B$; melhorar a qualidade da alimentação e uso dos Sistemas de Informação; institucionalizar uma cultura de avaliação da AB no SUS e de gestão com base na indução e acompanhamento de processos e resultados; estimular o foco da AB no usuário ${ }^{1}$.

Tendo em vista tratar-se de proposta ainda em implantação e devido à introdução de alguns aspectos e dispositivos que pretendem se constituir em inovações para a efetivação da PNAB no território nacional, este artigo teve por objetivo trazer uma reflexão sobre o PMAQ-AB como estratégia atual de avaliação dos serviços de atenção básica utilizada pelo MS, destacando, numa breve e preliminar análise, alguns de seus aspectos mais relevantes, tendo como norte a efetivação da implementação do SUS como política social voltada para o acesso universal e a equidade em saúde, por meio da descentralização e fortalecimento dos níveis municipais e locais de saúde.

\section{O PMAQ-AB COMO EstratÉGIA DE Avaliação}

Do ponto de vista das matrizes teórico-metodológicas que orientam as propostas de avaliação em saúde, parece ser consensual, já há algumas décadas, o caráter de imprevisibilidade e incerteza que marcam os problemas de saúde de uma população, e, portanto, também afetam as ações voltadas para a sua resolução. Na implementação dos programas e estratégias que compõem a Política Nacional de Saúde, a questão da avaliação veio ganhando especificidades, demarcando paulatinamente o papel da instância Federal como a principal formuladora dos processos de avaliação junto às Unidades Federativas e municípios.

O marco das Normas Operacionais Básicas, emitidas na década de 90 , veio sedimentar a prática da busca do consenso entre os níveis de execução político-administrativa do SUS por meio da proposição de instâncias e processos de pactuação, hoje integrados ao cotidiano da implementação dessas políticas, e utilizando-se de indicadores e metas referidos às principais questões de saúde pública. Isto não significa, no entanto, que tenha ocorrido efetiva institucionalização, pelos municípios, de práticas avaliativas.

Quando se trata do nível da AB, cujo programa prioritário é a atual estratégia saúde da família (ESF), há uma implicação importante, que é a que se explicita pela proposição de uma mudança de modelo assistencial, que resumidamente pode ser entendida pela ideia de um deslocamento, da ênfase em ações curativas, de caráter individual e voltadas para diagnóstico e tratamento de doenças, conferindo centralidade ao trabalho médico, para ações de caráter interdisciplinar e interprofissional, que incluam a identificação e o enfrentamento de determinantes sociais e biológicos, de fatores de risco, além das ações de recuperação e reabilitação ${ }^{6}$.

Esta mudança estaria a exigir transformações expressivas nas práticas e ações de saúde, nas relações entre atores sociais envolvidos, nos níveis da gestão municipal. Alguns autores ${ }^{7}$ entendem que indicadores e metas quantitativas não são capazes de apreender as mudanças qualitativas necessárias para a efetivação das mudanças, tornando-se necessário o desenvolvimento de uma cultura institucional de avaliação em saúde nos municípios.

\section{Avançando a institucionalização...}

Uma tentativa importante de fazer avançar a institucionalização foram os Estudos de Linha de Base do Projeto de Expansão e Consolidação do Saúde da Família (ELB/PROESF) desenvolvidos em meados da década de 2000, por meio de estudos de metaavaliação em municípios brasileiros, destacando três dimensões: a Político-institucional, a da Organização da Atenção e a do Cuidado Integral. Resultados desses estudos evidenciam a baixa cultura avaliativa nos municípios, questão agravada por problemas como rotatividade de gestores e profissionais, influências políticas locais, baixa qualificação profissional, entre outros $^{7}$. No âmbito das práticas das equipes, outra análise do ELB-PROESF evidenciou que o foco destas ainda se dava em torno de ações individuais e, quando coletivas, em torno de ações programáticas por agravos 
- hipertensão/diabetes, tuberculose, dentre outros ${ }^{8}$. Evidencia-se, portanto, que a implantação da ações avaliativas não depende apenas da definição de indicadores, e que uma abordagem pedagógica adequada pode colaborar para ampliar a capacidade avaliativa dos atores envolvidos?.

Em que pese esse conjunto de dificuldades, a ESF, modelo de atenção à saúde adotado como estratégia no Brasil, trouxe inegável aumento no acesso à saúde, entendido a partir da cobertura de serviços. Tal ampliação fomentou a necessidade de qualificação dos serviços ofertados aos usuários no nível primário de atenção, fazendo-se necessário o desenvolvimento de novas ações avaliativas para a tomada de decisão no nível da gestão do sistema de saúde 5 .

\section{Diretrizes}

Uma das principais diretrizes atuais do MS é executar a gestão pública com base na indução, monitoramento e avaliação de processos e resultados mensuráveis, garantindo acesso e qualidade da atenção em saúde a toda a população. Nesse sentido, diversificados esforços têm sido empreendidos no sentido de ajustar as estratégias previstas na PNAB na direção de reconhecer a qualidade dos serviços de $\mathrm{AB}$ ofertados à sociedade brasileira e estimular a ampliação do acesso e da qualidade nos mais diversos contextos existentes no país ${ }^{10}$, sendo o PMAQ-AB o mais recente.

As diretrizes do PMAQ-AB, são as seguintes: construir parâmetro de comparação entre as EAB; estimular processo contínuo e progressivo de melhoramento dos padrões e indicadores de acesso e de qualidade; transparência em todas as suas etapas; envolver, mobilizar e responsabilizar os gestores federal, estaduais, do Distrito Federal e municipais, as equipes de saúde de atenção básica e os usuários; desenvolver cultura de negociação e contratualização; estimular a efetiva mudança do modelo de atenção, o desenvolvimento dos trabalhadores e a orientação dos serviços em função das necessidades e da satisfação dos usuários; e demarcar o caráter voluntário para a adesão tanto pelas $\mathrm{EAB}$ quanto pelos gestores municipais ${ }^{2}$.

Sobre estes objetivos e diretrizes, vale destacar seu caráter amplo e abstrato em muitos aspectos, que trazem alguns questionamentos: qual seria a estratégia adotada para permitir a transparência das ações governamentais? Em que medida as comparações entre as EAB não estariam estimulando a competitividade entre as mesmas? Em que consiste, afinal, a tão proferida mudança de modelo de atenção? Qual a garantia que a adesão das EAB e Municípios ocorrem de modo voluntário?

O MS reconhece que essa mudança não está livre de entraves, portanto destaca alguns dos desafios que o PMAQ se propõe a enfrentar para garantir a qualificação da atenção primária: Precariedade da rede física; Ambiência pouco acolhedora das UBS; Inadequadas condições de trabalho para os profissionais; Necessidade de qualificação dos processos de trabalho das equipes de AB; Instabilidade das equipes e elevada rotatividade dos profissionais; Incipiência dos processos de gestão centrados na indução e acompanhamento da qualidade; Sobrecarga das equipes com número excessivo de pessoas sob sua responsabilidade; Pouca integração das equipes de $\mathrm{AB}$ com a rede de apoio diagnóstico e terapêutico e com os outros pontos da Rede de Atenção à Saúde (RAS); Baixa integralidade e resolutividade das práticas; Financiamento insuficiente e inadequado da $A B^{10}$. Apesar da menção desses muitos desafios em sua publicação original, não se percebe nem é proposta uma real reflexão sobre os determinantes dessas condições.

\section{Estrutura}

O PMAQ está estruturado em quatro fases complementares distintas, que configuram um ciclo continuo de melhoria do acesso e da qualidade da $\mathrm{AB}$ :

- Fase 1 - Adesão e contratualização: é a etapa formal de adesão ao programa, por meio da contratualização de compromissos e indicadores a serem estabelecidos entre as equipes de atenção básica e a gestão municipal, e desses com o Ministério da Saúde num processo que envolve pactuação regional e estadual e a participação do controle social.

- Fase 2 - Desenvolvimento: é a etapa central do programa, visto que é onde ocorre a operação da mudança desempenhada fundamentalmente pelas equipes de atenção básica e gestão municipal. Esta fase tem por foco as estratégias de autoavaliação e monitoramento das ações ${ }^{11}$. Como instrumento de autoavaliação o MS adaptou o modelo Avaliação para Melhoria da Qualidade da Estratégia Saúde da Família (AMQ) e também outras ferramentas de avaliação de serviços de saúde, utilizadas e validadas nacional e internacionalmente, a exemplo do MoniQuor, PCATool e Quality book of Tools ${ }^{12}$.

- Fase 3 - Avaliação externa: onde serão realizadas um conjunto de ações para verificar as condições de acesso e de qualidade dos municípios e equipes de atenção básica. É nesta fase que são apanhadas um conjunto de informações sobre as condições de acesso e de qualidade das EAB, o que possibilitará a certificação das equipes participantes do programa. A partir desta avaliação se reconhece e valoriza os resultados e esforços alcançados pelas $\mathrm{EAB}$ e gestores municipais no processo de qualificação da $\mathrm{AB}^{11}$.

- Fase 4 - Recontratualização: deve acontecer após a certificação das EAB. A partir da avaliação de desempenho de cada equipe, uma nova contratualização de compromissos e indicadores deverá ocorrer, completando assim o ciclo de qualidade esperado pelo programa ${ }^{10}$. 
É nesse processo de certificação das equipes que as estratégias para sua obtenção acabam por enfatizar a competitividade por melhores resultados, sendo financeiramente premiadas aquelas que apresentarem indicadores mais próximos das metas pactuadas. Ora, já há estudos suficientes que evidenciam que a sobrecarga de trabalho de agentes comunitários de saúde (ACS) e enfermeiros, descritas a partir da pressão da gestão pela necessidade de cumprimento de metas, se desdobram em situações de desgaste e sofrimento psíquico, isto apenas para mencionar duas categorias profissionais ${ }^{13,14}$. Torna-se necessário, portanto, problematizar e questionar em que medida a imposição de processos competitivos poderá colaborar para a melhoria da qualidade da atenção, e qual o custo desses processos sobre o bem estar dos trabalhadores de saúde e o respeito à sua autonomia profissional.

\section{Instrumentos avaliativos}

Outra questão a ser destacada refere-se à necessidade de novos instrumentos avaliativos. Entre as escassas publicações que suscitam reflexões e críticas à PMAQ- $A B$, ressalta-se o questionamento sobre a criação e avaliação por esse instrumento:

Do ponto de vista técnico, uma vez que já existem instrumentos consagrados e validados como a AMQ e o Primary Care Assessment Tool (PCATool), fica difícil entender a necessidade de se criar um novo instrumento, ainda que baseado em instrumentos pré-existentes. Os próprios indicadores de saúde do PMAQ-AB são muitas vezes questionáveis, como, por exemplo, o coeficiente de preventivos de câncer de útero realizados em mulheres de 15 anos ou mais - o INCA (Instituto Nacional de Câncer) recomenda o rastreamento apenas em mulheres de 25 aos 64 anos de idade; e colher um preventivo de seis em seis meses numa mulher nunca deveria ser equiparado a colher um preventivo de 3 em 3 anos em 6 mulheres. Mas a maior questão é: que conjunto de instituições de ensino será capaz de verificar a qualidade de todas as dezenas de milhares de equipes do País ${ }^{15: 7}$.

O Instrumento de Avaliação da Atenção Primária (PCATool - Primary Care Assessment Tool) foi criado na Johns Hopkins Primary Care Policy Center ${ }^{16,17}$ e elaborado com base no modelo de avaliação da qualidade de serviços de saúde proposto por Donabedian ${ }^{18}$. Apresenta originalmente versões autoaplicáveis destinadas a crianças, adultos maiores de 18 anos, profissionais de saúde e, também, ao coordenador / gerente do serviço de saúde ${ }^{19}$.

Este instrumento mede a presença e a extensão dos quatro atributos essenciais (acesso de primeiro contato do indivíduo com o sistema de saúde; longitudinalidade; integralidade; coordenação da atenção) e dos três atributos derivados da APS: Atenção à saúde centrada na família, orientação familiar; orientação comunitária; competência cultural ${ }^{17,19}$.
Este modelo de avaliação baseia-se na avaliação de aspectos de estrutura, processo e resultados dos serviços de saúde. Assim sendo, devido a ausência de ferramentas para medir essas interações entre os usuários e os profissionais mediados pela estrutura do serviço de saúde no contexto da APS em nosso país, o PCATool preencheria uma lacuna, promovendo medida de base individual sobre a estrutura e, principalmente, o processo de atenção em APS ${ }^{19}$.

O PCATool foi adaptado à realidade brasileira, cada versão do instrumento foi modificada

em uma ferramenta aplicável por meio de entrevistadores e passou por um processo de tradução e tradução reversa, adaptação, debriefing e validação de conteúdo e de construto, além da análise de confiabilidade $^{19: 10}$.

Apesar desses esforços, o PCATool não foi amplamente utilizado no Brasil.

Retomando o pensamento de Fontenelle ${ }^{15}$, cujo estudo data de pouco mais de 6 meses depois da instituição da Portaria n ${ }^{\circ} 1.6542$, o autor relata certa incerteza quanto à utilização do PMAQ- $\mathrm{AB}$, visto que até aquela ocasião ainda não eram conhecidos os instrumentos de autoavaliação e de avaliação externa a serem utilizados. Ao mesmo tempo, destaca que a avaliação sistemática da APS trás a possibilidade de ratificar a superioridade da ESF. Além disso, por conta de uma participação mais significativa do enfermeiro e a obrigatoriedade da presença do ACS, a atenção primária à saúde (APS) deverá se assemelhar cada vez mais ao preconizado na ESF. $O$ autor ainda concluiu que, apesar das vantagens, é estranho que o MS não tenha adotado um instrumento de avaliação já validado e abrangente como o PCATool.

Por fim, vale ainda problematizar o expresso caráter de adesão voluntária à PMAQ-AB pelos municípios, questionando a real possibilidade de escolha para a adesão, dada a ainda centralidade político-decisória da política de saúde brasileira, no que se efetivou como descentralização executória das ações de saúde, mas ainda dependentes do financiamento federal e da condução técnica e política por parte do MS.

\section{Avaliação de política pública}

A atenção primária em saúde (APS), constituiuse, desde a década de 80 , na principal estratégia de ampliação do acesso a um conjunto básico de serviços de prevenção e promoção da saúde, com foco em países pobres e/ou emergentes, como o Brasil ${ }^{20}$, financiados por órgãos internacionais que, no marco das reformas de estado da década de 90 , induziram fortemente a ampliação da oferta de ações e serviços de saúde. O campo no qual estas políticas vem se concretizando, no entanto, é também o de disputa entre projetos políticos e marcos conceituais sobre o que é ou deve ser o nível da APS, por sua vez um conceito com fluidez e polissemia suficiente para permitir apropriações e interpretações diversas ${ }^{21}$. 
Sobre o PMAQ-AB, vale ressaltar alguns aspectos que parecem exercer forte influência na configuração mais recente dos modos de implantação da ESF na AB. O Programa, implantado em 2011 pela Portaria 16442, segue-se à publicação da nova $\mathrm{PNAB}^{1}$, de outubro de 2011. De forma geral, a nova PNAB manteve a essência da anterior e incorporou a flexibilidade de carga horária para médicos e a implantação dos NASF, entre outros dispositivos. O PMAQ-AB estabelece parâmetros de avaliação definidos, a serem implementados por meio de fases, que incluem desde processos de autoavaliação pelos sistemas municipais, até a avaliação externa. Os resultados obtidos nessa avaliação podem se desdobrar em incentivos financeiros para os fundos municipais de saúde, de acordo com o desempenho demonstrado.

Estabelece-se, dessa forma, uma aproximação entre esse processo de avaliação de política pública e o que vem sendo denominado de Novas Políticas de Recursos Humanos - aquelas que se pautam por conceitos e estratégias de gerenciamento da qualidade total, gerenciamento participativo, entre outras denominações ${ }^{22}$.

O PMAQ é complexo e recente não tendo conseguido ainda implementar as estratégias de mudança das realidades ${ }^{23}$.

\section{Conclusão}

Apesar de sua recente implantação, o PMAQ$\mathrm{AB}$ tem sido questionado também no que tange aos demais processos de trabalho nas equipes. Sendo uma das principais políticas do MS, aspectos de estímulo à relação competitiva entre equipes e à obtenção do reconhecimento por mérito, essencialmente relacionados à quantificação da produção e aos indicadores de resultados, com ênfase na diminuição de doenças, levam a questionar em que medida não se está avançando na direção de uma saúde por resultados, em lugar de reafirmar o direito universal à saúde integral.

Atualmente o PMAQ encontra-se em seu segundo ciclo, o que é considerado muito precoce, visto que todo o processo nacional é bastante complexo, dispendioso e exaustivo, tanto para as EAB que são avaliadas quanto para a as equipes de avaliadores. Além disso, é um tempo demasiadamente curto para identificação de problemas, definição de estratégias de intervenção e mudança de realidades. O segundo ciclo teve início sem que as $\mathrm{EAB}$ tenham, de fato, conseguido contratualizar e implementar estratégias de mudanças.

\section{REFERÊNCIAS}

1.Ministério da Saúde (Br). Portaria no 2.488, de 21 de outubro de 2011. Aprova a Política Nacional de Atenção Básica [...]. Brasília (DF): Gabinete Ministerial; 2011. 2.Ministério da Saúde (Br). Portaria no 1.654, de 19 de julho de 2011. Institui, no âmbito do Sistema Único de
Saúde, o Programa Nacional de Melhoria do Acesso e da Qualidade da Atenção Básica (PMAQ-AB [...]. Brasília (DF): Gabinete Ministerial; 2011.

3.Pinto HA. Múltiplos olhares sobre e a partir do Programa Nacional de Melhoria do Acesso e Qualidade [dissertação de mestrado]. Porto Alegre (RS): Universidade Federal do Rio Grande do Sul; 2014.

4.Pinto HA, Souza ANA, Ferla AA. The National Program for Access and Quality Improvement in Primary Care: faces of an innovative policy. Saúde em debate. 2014; 38 (esp.):358-72.

5.Fausto MCR, Fonseca MHS, organizadores. Rotas da atenção básica no Brasil: experiências do trabalho de campo PMAQ-AB. Rio de Janeiro: Saberes editora; 2013. 6.Paim JS. A reforma sanitária e os modelos assistenciais. In: Silva LMV, organizadores. Saúde coletiva. Salvador (BA): Centro Editorial e Didático, Universidade Federal da Bahia; 1994. p. 61-82.

7.Camargo KRJ, Campos SEM, Teixeira MTB, Mascarenhas MTM, Mauad NM, Franco TB, David HMSL, Alves MJM, Ribeiro LC. Vivências e reflexões de avaliação na atenção básica: a experiencia dos ELBs/Proesf em Minas Gerais e no Espírito Santo. In: Hartz Z, Felisberto E, Silva LMV, organizadores. Meta-análise da atenção básica à saúde. Rio de Janeiro: FIOCRUZ; 2008. p. 119-52.

8.Tumé E, Soares MU, Dilélio AS. Projetos e ações inovadoras na atenção primária à saúde. In: Soares CB, Campos CMS, organizadores. Fundamentos de saúde coletiva e o cuidado de enfermagem. São Paulo: Ed. Manole; 2013. p.349-68.

9.David HMSL, Camargo Jr KR. "Prática avaliativa na atenção básica: revisitando a pedagogia da problematização. Rev enferm UERJ. 2013; 21: 677-81.

10.Ministério da Saúde (Br). Secretaria de Atenção à Saúde. Programa Nacional de Melhoria do Acesso e da Qualidade da Atenção Básica (PMAQ): manual instrutivo. Brasília (DF): Ministério da Saúde; 2011.

11.Pinto HA, Sousa A, Florêncio AR. O Programa Nacional de Melhoria do Acesso e da Qualidade da Atenção Básica: reflexões sobre o seu desenho e processo de implantação. RECIIS - R Eletr de Com Inf Inov Saúde. 2012; 6 (2).

12.Ministério da Saúde (Br). Secretaria de Atenção à Saúde. Autoavaliação para a Melhoria do Acesso e da Qualidade da Atenção Básica : AMAQ. Brasília (DF): Ministério da Saúde; 2012.

13.Carreiro GSP, Ferreira Filha MO, Lazarte R, Silva AO, Dias D. O processo de adoecimento mental do trabalhador da Estratégia Saúde da Família. Revista Eletrônica de Enfermagem. 2013; 15: 146-55.

14.Rosa AJ, Bonfanti AL, Carvalho CS. O sofrimento psíquico de agentes comunitários de saúde e suas relações com o trabalho. Saúde e Sociedade. 2012; 21 (1): 141-52. 15.Fontenelle LF. Mudanças recentes na Política Nacional de Atenção Básica: uma análise crítica. Rev bras med fam comunidade. 2012; 7 (22): 5-9.

16.Starfield B, Cassady CE, Hurtado MP, Berk RA, Nanda JP, Friedenberg LA. Measuring consumer experiences with primary care. Pediatrics (United States). 2002; 105: 998-1003. 
17.Starfield B, XU J, SHI L. Validating the adult primary care assessment tool. The Journal of Family Practice (United States). 2001; 50: 161-75.

18.Donabedian A. Evaluating the quality of medical care, 1966. The Milbank quarterly (United States). 2005; 83: 691-729.

19.Ministério da Saúde (Br). Secretaria de Atenção à Saúde. Departamento de Atenção Básica. Manual do instrumento de avaliação da atenção primária à saúde: primary care assessment tool pcatool - Brasil. Brasília (DF): Ministério da Saúde; 2010.

20.Ronzani TM, Silva CM. Brazil's Family Health Program according to healthcare practitioners, managers and users. Ciênc saúde coletiva. 2008; 13: 23-34.

21.Paim J. Atenção Primária à Saúde: uma receita para todas as estações? Saúde em Debate. 2012; 36: (94): 343. 22.Moraes PN, Iguti AM. Avaliação do desempenho do trabalhador como forma peculiar de prescrição do trabalho: uma análise do PMAQ-AB. Saúde em Debate. 2013; 37 (98): 416-26.

23.Fausto MCR, Mendonça MHM, Giovanella L. Experiências de avaliação da atenção básica no Brasil. In: Fausto MCR, Fonseca HMS, organizadores. Rotas da atenção básica no Brasil: experiências do trabalho de campo Pmaq AB. Rio de Janeiro: Saberes editora; 2013. p. 288-309. 